\title{
Follow the BAT: Monitoring Swift BAT FoV for Prompt Optical Emission from Gamma-ray Bursts
}

\author{
T. N. Ukwatta*, J. Linnemann*, K. S. Dhuga ${ }^{\dagger}$ and N. Gehrels** \\ ${ }^{*}$ Michigan State University, Physics and Astronomy Department, East Lansing, MI 48824, USA \\ ${ }^{\dagger}$ The George Washington University, Washington, D.C. 20052, USA \\ **NASA Goddard Space Flight Center, Greenbelt, MD 20771, USA
}

\begin{abstract}
We investigate the feasibility of implementing a system called 'Follow the BAT' that will coordinate ground-based robotic optical and near infrared (NIR) telescopes to monitor the Swift BAT field-of-view (FoV). The system will optimize the monitoring locations in the BAT FoV based on individual robotic telescopes' location, FoV, sensitivity and local weather conditions. The aim is to perform coordinated BAT FoV monitoring by professional as well as amateur astronomers around the world. The scientific goal of the proposed system is to facilitate detection of prompt optical and NIR emission from GRBs, especially from short duration GRBs. We have performed a Monte Carlo simulation to investigate the feasibility of the project.
\end{abstract}

Keywords: Gamma-Ray Bursts

PACS: $95.75 . \mathrm{Rs}$

\section{INTRODUCTION}

Our understanding of Gamma-ray Bursts (GRBs) progressed very rapidly after the detection of multi-wavelength afterglows. Usually, a favorably positioned GRB gets fairly good multi-wavelength afterglow coverage. However, multi-wavelength observations of the prompt emission are sparse. The reason is obvious. The location and the time of a GRB cannot be predicted, and currently, it is not possible to monitor the whole sky using multi-wavelength instruments. Despite this fact, multi-wavelength observations of prompt emission of GRBs are crucial to enhance our understanding of GRB physics.

Initial detection of prompt optical emission from GRB 990123 [1] has been followed by few bursts that are observed in optical wavelengths during the gamma-ray emission. Some of these detections are due to fortunate simultaneous observations from multiple detectors (e.g. GRB 080319B; [2]) or when the GRB was preceded by a strong precursor event (e.g. GRB 061121; [3]). In addition, a number of fast slewing robotic optical telescopes such as ROTSEIII [4], TAROT [5], REM [6], and RAPTOR [7] have been used to catch prompt optical emission while the gamma-ray emission is still ongoing. However, all these fast slewing telescopes are at the mercy of the propagation time of the burst notice from the space craft to the telescope and also on the slewing time of the telescope. There has never been a systematic coordinated effort to detect prompt optical emission from GRBs independent of the notice propagation time and the slewing time.

Even though a few cases of prompt optical detections have been reported involving long duration bursts, so far there has been no detection of prompt optical emission from short duration bursts. If the progenitors of short duration bursts are merging compact objects, then there are theoretical predictions of possible early optical/UV emission from an accompanying 'mini-supernove' [8], caused by the ejection of radioactive material from the merging system. This optical signal fades away very quickly and can only be detected by monitoring a nearby short burst location at the burst onset.

Based on the current sample of bursts with prompt optical coverage, it seems there are two categories of GRBs. The first category is characterized by bright optical transients that are uncorrelated with the gamma-ray emission (e.g. GRB 990123; [1]). The second category has weak optical emission correlated with the gamma-ray emission (e.g. GRB 041219A; [9]). The optical emission of the first category is thought to originate from reverse shocks [10] whereas for the second category optical emission is generally interpreted as the low-energy tail from the gamma-ray emission [11]. However, interpretation of the second category is questionable after detection of bright correlated optical emission from GRB 080319B.

Another interesting question is whether GRB precursors are associated with any optical or NIR emission. Currently there are no observational data on precursors other than on gamma-ray wavelengths. Evidence for precursors has been 
reported both in short [12] and long [13,14] duration bursts. There are number of interpretations for GRB precursors such as 1) photospheric blackbody emission just before the transition to optically thin regime, 2) due to interaction of the relativistic jet with the stellar envelope, or 3) due to two-step collapse of the central engine. Detection of optical emission coincident with a gamma-ray precursor event may cause us to rethink these interpretations.

Currently, there are robotic telescopes that can slew to the location of the burst as fast as $\sim 5$ seconds after the receipt of the GCN notice [15]. However, the time difference between the trigger time and the arrival time of the GCN notice is significant. Typically this delay can be $\sim 15$ seconds. If one wants to observe prompt optical emission from bursts which last for less than $\sim 20$ seconds, especially short bursts, then one way to observe them is by monitoring the BAT FoV.

\section{PROPOSED OBSERVING PROGRAM}

Inspired by successful 'citizen science' projects such as Galaxy Zoo and NASA's Stardust@Home projects, we propose to implement a system called 'Follow the BAT' to facilitate monitoring of the BAT FoV for prompt optical and NIR emission from GRBs. This observing program could be specially aimed at amateur astronomers around the world. Proliferation of amateur robotic telescopes with high quality CCD cameras have opened a new avenue to study prompt optical emission from GRBs. The basic objective of the 'Follow the BAT' system is to coordinate large number of ground based robotic telescopes to monitor different patches of the BAT FoV. Selection of various patches in the BAT FoV will be done based on the number of available telescopes, individual robotic telescopes' location, FoV, sensitivity and local weather conditions.

Some of the important observational questions that can be potentially answered by such an observing program are given below:

- Is there any prompt optical emission from short duration GRBs?

- Are GRB precursors associated with any simultaneous optical emission?

- What fraction of GRBs have prompt optical emission at the trigger time?

- How is the prompt optical light curve correlated with the gamma-ray light curve? Are there two populations?

- What are the variability time scales of the GRB prompt optical and NIR emission?

The proposed system is envisioned to have a web interface which will allow people to register their robotic telescopes and obtain useful information such as 1) the BAT pointing direction in real-time, 2) all participating robotic telescopes, 3) regions of the BAT FoV allocated to specific robotic telescope, and 4) covered and uncovered regions of the BAT FoV at a given time.

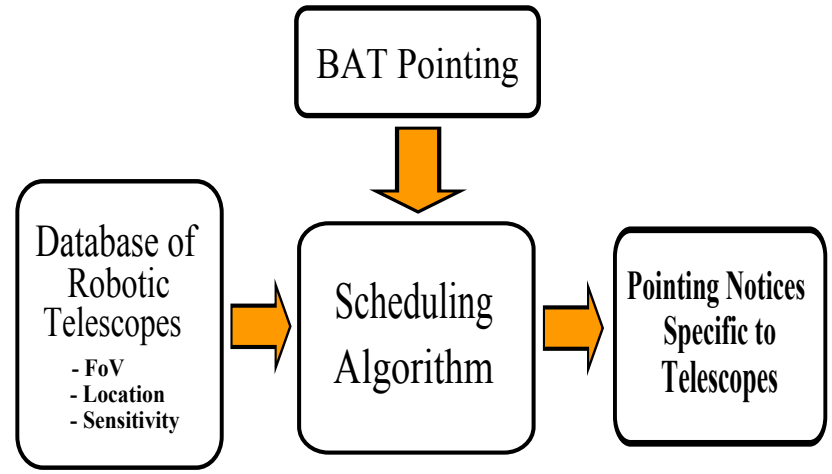

FIGURE 1. Schematic block diagram of the proposed 'Follow the BAT' software system.

Unlike the GCN system [15] which sends notices to large number of recipients, the proposed system will send customized targeted messages to individual registered robotic telescopes. The individual messages will be sent via email or socket connections. These customized messages will have assigned pointing locations for the robotic telescope. Another advantage for the telescopes that are pointing inside the BAT FoV is that even when they do not necessarily catch the burst they are relatively close by to the burst location and would therefore be able to slew to it relatively quickly. Hence, by participating in the 'Follow the BAT' program, robotic telescopes can potentially increase their response times for burst triggers. 
A schematic block diagram of the proposed 'Follow the BAT' software system is shown in the Figure 1. The scheduling algorithm will check the BAT FoV observability of each robotic telescope and assign them to different parts of the BAT FoV. In doing this the algorithm will consider number of parameters such as individual telescopes' FoV and sensitivity. In addition, it will also consider the bore-sight angle distribution of previously detected Swift bursts.

\section{FEASIBILITY AND CHALLENGES}

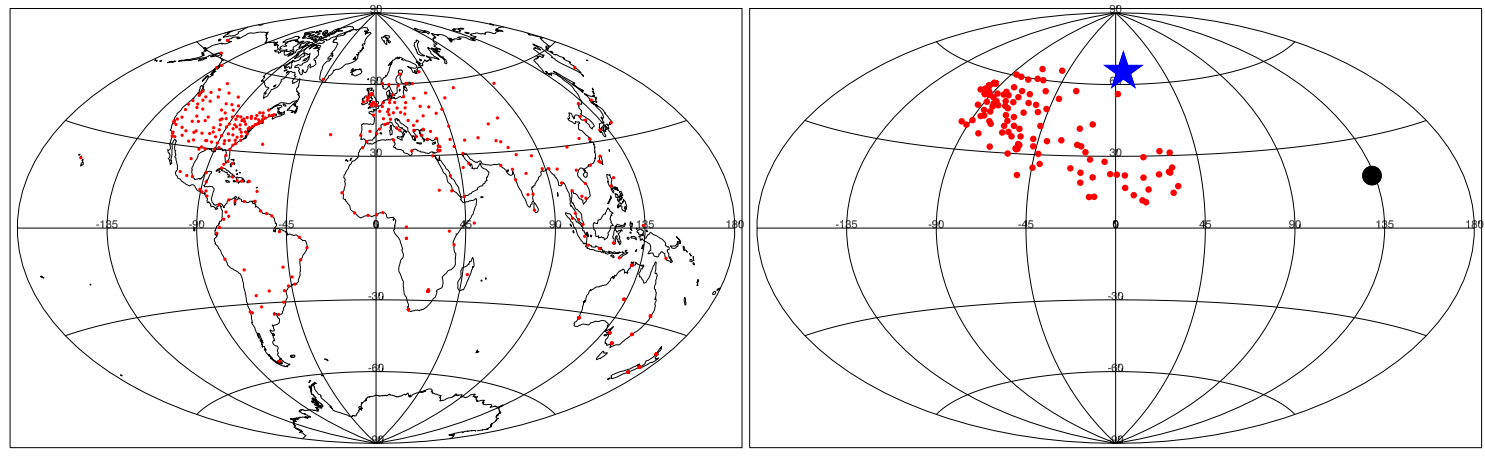

FIGURE 2. Left Panel: Aitoff projection of the world map showing the distribution of telescopes used in the simulation. Right Panel: Galactic Aitoff projection of the location of the burst (blue star), the Sun (black filled circle) and zenith coordinates of telescopes that can observe the burst (red circles).

In order to investigate the feasibility of the project, we performed a Monte Carlo simulation to study the probability of detection of prompt optical emission from Swift GRBs. We assumed that Swift detects about 100 GRBs per year distributed isotropically in the sky and also throughout the year. We have distributed robotic telescopes in such a way that they roughly trace the major cities in the world as shown in the left panel of Figure 2. Then for each burst we tracked the path of the Sun and selected a set of telescopes away from the Sun and within few hours from the burst location to monitor the BAT FoV. The right panel of Figure 2 shows the selected set in red circles for a given burst. We calculated the probability of detecting a given burst by summing FoV solid angles of all telescopes in the selected set and then dividing by the BAT FoV solid angle $\left(60.0^{0} \times 60.0^{0}\right)$. We repeated this procedure for every simulated burst, while changing the number of total telescopes available and $\mathrm{FoV}$ of telescopes.
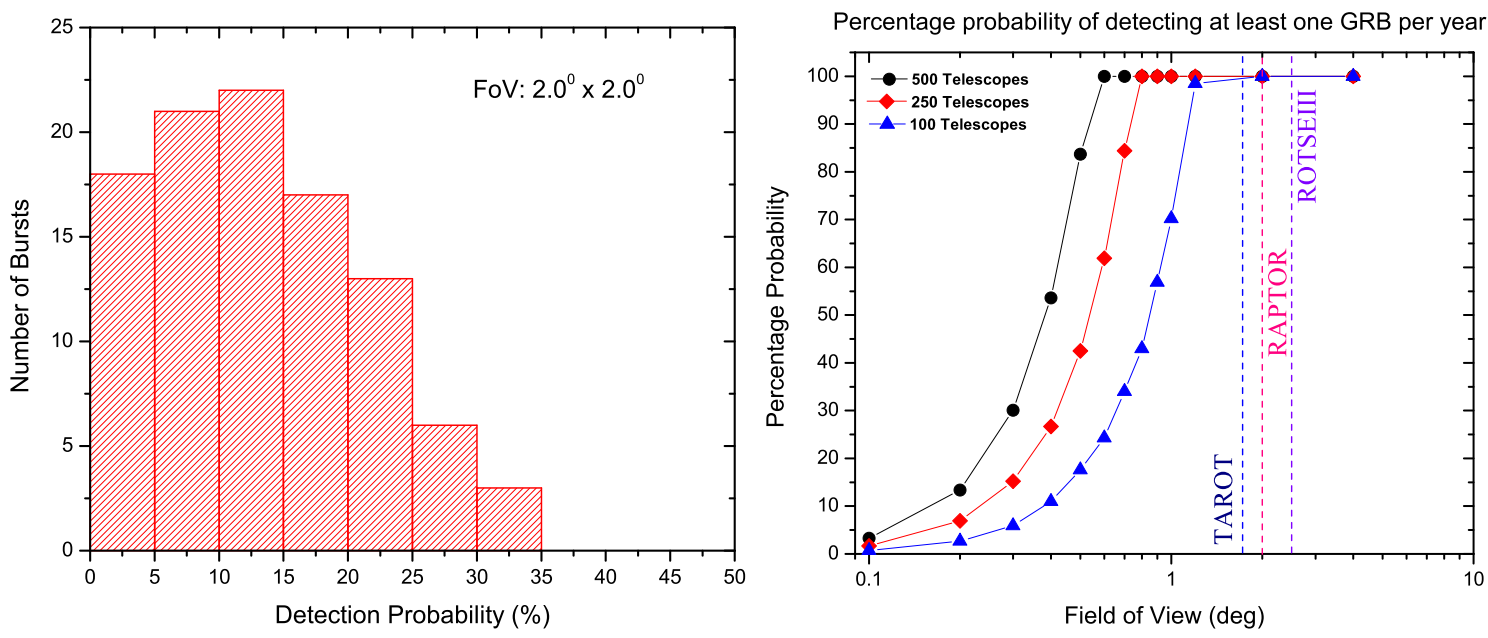

FIGURE 3. Left Panel: Histogram of detection probability of 100 bursts with 500 telescopes with FoV of $2.0^{0} \times 2.0^{0}$. Right Panel: The percentage probability of detecting at least one burst per year as a function of the FoV when 100, 250, and 500 telescopes are participating in the program. 
The results of our simulation are shown in Figure 3. For these particular simulations we have assumed all the robotic telescopes have the same FoV. The number of participating telescopes in the observing program are taken to be 100, 250 or 500 . The left panel of Figure 3 shows the distribution of percentage probability values of detecting bursts with 500 telescopes with FoV of $2.0^{0} \times 2.0^{0}$.

The right panel of Figure 3 depicts the percentage probability of detecting at least one burst per year as a function of the FoV. With 500 telescopes participating, it is possible to detect at least one burst using robotic telescopes with FoV of $0.6^{0} \times 0.6^{0}$. This value is close to the value of a typical $\mathrm{FoV}$ of an amateur robotic telescope. It is also interesting to note that if we have about 100 telescopes participating with $\mathrm{FoV}$ of $1.0^{0} \times 1.0^{0}$, we can detect at least one burst per year. The simulation did not take into account the moon fraction and fraction of the time BAT FoV is covered by clouds.

In practice, however, there are number of serious challenges to carry out this type of observing program:

- Number of telescopes needed to perform the observation is relatively large.

- Number of telescopes required can be brought down by increasing the FoV. However, the increment of the FoV may result in loss of sensitivity.

- Currently, the number of amateur robotic observatories capable of monitoring BAT FoV is small. However, this number is growing steadily.

- Professional observatories which are capable of monitoring the BAT FoV have dense observation schedules and are unlikely to participate in the program.

Despite these difficulties, 'Follow the BAT' type of observing program could enable the investigation of the optical properties of the prompt emission of GRBs, particularly in short ones. It could also enable the study of the precursors in optical wavelengths for the first time. In addition, the project will potentially allow and attract the participation of amateur astronomers and their telescopes around the world.

\section{DISCUSSION AND CONCLUSION}

In this paper we have investigated the feasibility of implementing an observation program to monitor the Swift BAT FoV for prompt optical and NIR emission. The simulation results show, even though there is a fair chance of detecting a few GRBs with prompt optical emission, the amount of effort needed to carry out the task is substantial. However, astonishing $\sim$ 5th magnitude prompt optical emission from GRB 030319B and the detection of 12 magnitude optical flare from very high redshift burst, GRB 050904 (with redshift of 6.29) indicate that we should be able detect optical emission from GRBs up to very high redshift with varying degrees of brightness. With moderate exposure times $(\sim$ seconds) amateur telescopes may be able to reach these magnitude levels and observe prompt optical emission from GRBs. The decades old observation model - space-based gamma-ray observatories discovering bursts and groundbased observers following - is approaching its limits after the highly successful Swift mission. Now may be the time to change this traditional observation model and try to observe GRBs in multi-wavelengths as they happen.

\section{REFERENCES}

1. Akerlof, C., et al. 1999, Nature, 398, 400

2. Racusin, J. L., et al. 2008, Nature, 455,183

3. Page, K. L., et al. 2007, ApJ., 663, 1125

4. Akerlof, C. W., et al. 2003, PASP, 115, 132

5. Klotz, A., Boër, M., Atteia, J. L., \& Gendre, B. 2009, AJ, 137, 4100

6. Zerbi, R. M., et al. 2001, Astronomische Nachrichten, 322, 275

7. Vestrand, W. T., Theiler, J., \& Woznia, P. R. 2004, Astronomische Nachrichten, 325, 477

8. Li, L.-X., \& Paczyński, B. 1998, ApJ. Lett., 507, L59

9. Vestrand, W. T., et al. 2005, Nature, 435, 178

10. Jin, Z. P., \& Fan, Y. Z. 2007, MNRAS, 378, 1043

11. Genet, F., Daigne, F., \& Mochkovitch, R. 2007, A.\& A., 471, 1

12. Troja, E., Rosswog, S., \& Gehrels, N. 2010, ApJ., 723, 1711

13. Koshut, T. M., et al. 1995, ApJ., 452, 145

14. Lazzati, D. 2005, MNRAS, 357,722

15. Barthelmy, S. D., et al. 1998, Gamma-Ray Bursts, 4th Hunstville Symposium, AIPC, 428, 99 